\title{
La circulation dans le liber
}

\author{
Circulation in the inner bark
}

\author{
Jean-Louis Bonnemain \\ Laboratoire de Biologie et Physiologie végétales \\ Station Biologique de Beau-Site, 25 Faubourg Saint-Cyprien, 86000 Poitiers
}

Le liber des plantes supérieures est formé de plusieurs types cellulaires : élément de parenchyme libérien horizontal ou rayon libérien, élément de parenchyme libérien vertical, cellule criblée, cellule compagne ou cellule de transfert et éventuellement fibre, collocyte ou laticifère. Sa fonction essentielle est d'assurer la circulation, vers les régions en voie de croissance ou accumulant des réserves, de substances trophiques variées, en particulier les produits de la photosynthèse. Il peut aussi accumuler des réserves ou avoir un rôle de soutien, notamment chez certaines espèces herbacées. Enfin, il assure au moins dans quelques cas la propagation d'excitations.

Chez les plantes supérieures, les cellules criblées dont le diamètre est de 20 à $40 \mu \mathrm{m}$ et dont la longueur est de quelques centaines de microns sont disposées bout à bout, constituant le tube criblé (fig. 1). Chaque cellule criblée est séparée de la suivante par le crible, une paroi

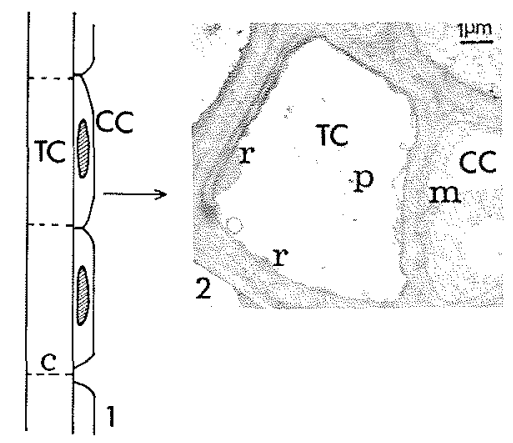

Figure 1 - Tube criblé (TC) bordé par des cellules compagnes (CC). Ches les végétaux évolués le crible (c) est perpendiculaire ou presque à l'axe du tube criblé.

Figure 2 - Tube criblé (TC) et cellule compagne (CC) de Plantago lanceolata vus en section transversale $(r$, réticulum sous ses deux formes caractéristiques ; $\mathrm{p}$, protéine- $\mathrm{P} ; \mathrm{m}$, mitochondrie) (avec la permission de M. Doffin). munie de pores (fig. 1,7 et 8 ) dont le diamètre oscille souvent de 0,5 à $5 \mu \mathrm{m}$. La surface des pores oscille en général entre le quart et la moitié de celle de l'ensemble du crible. C'est à l'intérieur du tube criblé que circulent les produits de la photosynthèse. Chaque cellule criblée est associée à une ou plusieurs cellules compagnes.

En section transversale, le rapport "surface des tubes criblés (partie interne)/surface des vaisseaux (partie interne)" est extrêmement variable, mais parfois de l'ordre de 0,1 chez les plantes herbacées. En admettant que les tubes criblés soient le siège d'un écoulement de sève, le débit dans le phloème est donc considérablement inférieur à celui du xylème, la vitesse de circulation n'étant pas supérieure dans le premier tissu.

Ultrastructure des cellules "nobles" du phloème (cellule criblée, cellule compagne et cellule de transfert)

La cellule criblée différenciée est une cellule vivante mais sans noyau et sans appareil vacuolaire classique. Sa différenciation est en effet accompagnée de régressions protoplasmiques spectaculaires (hypertrophie et disparition du noyau, disparition du tonoplaste, des microtubules, des ribosomes, du golgi) et du percement des pores du crible. Chez les Dicotylédones, elle est aussi marquée, et cela très précocement, par l'apparition d'inclusions cytoplasmiques particulières, "les corps muqueux". Leur nombre et leur forme varie avec les espèces. Chez le tabac, la future cellule criblée possède un seul et volumineux corps muqueux. Ce dernier est constitué de tubules protéiques dont le diamètre est d'environ $230 \AA$ : il s'agit de protéine- $P_{1}$ (Cronshaw et Esau, 1967 et 1968).

Dans la cellule criblée fonctionnelle (Fig. 2), le cytoplasme se trouve réduit à un très mince liséré tapissant la 
paroi ; on reconnaît des éléments du réticulum et, selon les espèces, des mitochondries ou des plastes dont le contenu varie avec les groupes végétaux. Les filaments protéiques provenant, selon la conception classique, de la dispersion des corps muqueux lors de la phase finale de la différenciation ou résultant, au moins en partie, d'une synthèse continue au niveau des cellules compagnes (Nuske et Eschrich, 1976) ont, selon les photos publiées par les cytologistes, une distribution très variable :

- ils peuvent apparaitre dispersés dans la lumière de la cellule criblée et dans les pores du crible avec une distribution assez homogène (Fig. 3) ;

- ils peuvent être groupés en faisceaux (Fig. 5) ;

- ils peuvent être particulièrement nombreux et serrés dans les pores, formant de véritables bouchons (Fig. 4 et 7) ;

- ils peuvent occuper une position pariétale, laissant vide la lumière de la cellule criblée et les pores (Fig. 6 et 8 ).
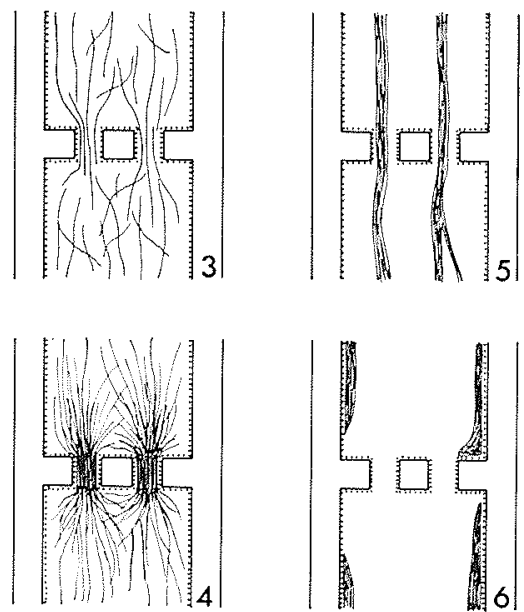

Figures 3-6 - Quelques distributions possibles des protéines-P de la cellule criblée chez les Dicotylédones (voir aussi les figures 11-14).

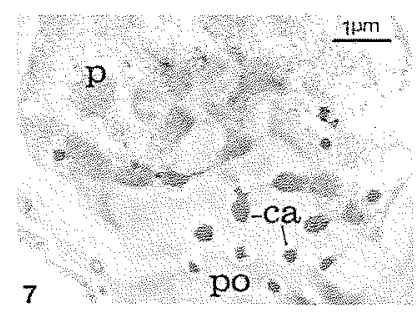

Figure 7 - Portion du crible du liber, ou phloème, de Plantago major, avec les pores (po) complètement obstrués par de la callose (ca) et des tubules de protéine-P (p) (avec la permission de $M$. Doffin). La présence d'une abondante callose est peutêtre due à une fixation trop lente du matériel et la présence de protéine-P dans les pores peut, elle-même, illustrer une réaction aux manipulations. Naturellement si la distribution des protéines-P était identique dans la cellule criblée fonctionnelle, il faudrait rejeter l'idée d'un mouvement par pression de turgescence et envisager d'autres mécanismes du transport libérien : glissements moléculaires le long des tubules de protéine-P, électro-osmose (comme le suppose Spanner, cf. Qureshi et Spanner, 1973), etc.

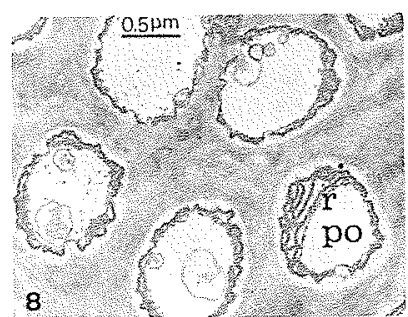

Figure 8 - Portion du crible du phloème de Curcubita maxima (Evert, Eschrich et Eichhorn, 1973, avec la permission de S.E. Eichhorn). Les pores (po), bordés par du réticulum (r) sont vides. Un écoulement de sève par pression de turgescence est possible.

Il est évident que ces informations sont du plus haut intérêt lorsqu'il s'agit de préciser s'il y a écoulement de sève dans la cellule criblée ou déplacements moléculaires sans mouvement d'eau. Malheureusement elles sont peu sûres, car la cellule criblée est une cellule très sensible et fragile, répondant par des modifications structurales à des agressions chimiques et physiques. Il est connu par exemple qu'une élévation de température, les ultra-sons, une blessure, provoquent une rapide biosynthèse de callose au niveau des pores. Par ailleurs, la fixation au glutaraldéhyde, couramment pratiquée, s'accompagne de modifications structurales et la plus importante serait, selon Fischer (1975), de provoquer la dispersion des inclusions protéiques ! Ce dernier point n'est cependant pas confirmé par des observations plus récentes.

La cellule compagne (Fig. 2), cellule-sœur de la cellule criblée, s'oppose à cette dernière par ses caractères cytologiques : noyau volumineux, cytoplasme dense, ribosomes nombreux, réticulum bien représenté, vacuome réduit et mitochondries relativement nombreuses. Chez les Gymnospermes, les cellules criblées sont associées à des cellules abuminifères.

Dans les fines nervures de nombreuses Dicotylédones herbacées, les cellules criblées sont associées à des cellules de transfert. Ces dernières se caractérisent principalement par l'abondance et la densité du cytoplasme, leur remarquable richesse en mitochondries et surtout par leur paroi présentant de nombreuses protubérances internes. Ces replis, augmentant considérablement le rapport $\frac{\text { surface }}{\text { volume }}$, doivent faciliter les échanges entre l'apoplaste et le symplaste.

\section{Composition de l'exsudat}

Diverses techniques sont utilisées pour connaître la nature des molécules transportées dans le phloème mais, depuis une vingtaine d'années, on fait appel aux aphidiens. Ces insectes, après quelques essais, plantent leurs stylets dans une cellule criblée. Les stylets, sectionnés près de la tête de l'insecte, font office de drain : de cette façon on peut recueillir, pour l'analyse, 1 ou $2 \mu \mathrm{l}$ d'exsudat libérien par drain et par heure ou par jour selon les cas. 
Assez exceptionnellement il suffit de faire une incision dans le phloème pour obtenir l'exsudat libérien. Cela se produit notamment chez les palmiers à sucre qui peuvent libérer, par arbre, une dizaine de litres de sève par jour. Cette sève contient environ 10 à $15 \%$ de sucre et devient le vin de palme après fermentation. Outre les glucides, en fait le saccharose associé éventuellement des oligoholosides comme le raffinose, stachyose, verbascose ou encore à des hexitols, l'exsudat libérien contient des acides aminés, des acides organiques, des nucléotides, en particulier de l'ATP, et de nombreux autres types moléculaires. L'exsudat libérien se caractérise en outre par une teneur relativement élevée en $K^{+}$ ( 1 à $10 \mathrm{~g} /$ litre) et une faible teneur en $\mathrm{Ca}^{++}$. Sa pression osmotique est souvent comprise entre 15 et 25 bars.

\section{Vitesse de transport}

On cite parfois l'estimation faite en 1922 par Dixon. Un tubercule de pomme de terre pesant $210 \mathrm{~g}$ et contenant $50 \mathrm{~g}$ de matières combustibles est rattaché à la plante par un stolon dont le liber a une section transversale de $0,0042 \mathrm{~cm}^{2}$. La concentration en sucre de l'exsudat libérien étant d'environ $10 \%$, le volume du liquide transporté doit être proche de $500 \mathrm{~cm}^{3}$ et cela en une centaine de jours. La vitesse du courant est donc de $\frac{500}{0,0042 \times 100 \times 24} \simeq 50 \mathrm{~cm} \mathrm{~h}^{-1}$ dans le stolon, selon l'auteur. Ce calcul doit être quelque peu amendé, notamment parce qu'une partie seulement du phloème (le $1 / 4$ environ) assure le transport, mais déjà la vitesse de $50 \mathrm{~cm} \mathrm{~h}^{-1}$ était apparue impossible à l'époque, car jugée beaucoup trop élevée. En fait, on sait maintenant qu'il s'agit d'une vitesse typique du transport libérien : par conséquent un écoulement de sève peut rendre compte de l'accumulation de glucides observée dans le tubercule de pomme de terre.

Selon les espèces et selon les stades du développement, la vitesse du transport libérien varie quelque peu, mais elle est en général comprise entre 3 et $300 \mathrm{~cm} \mathrm{~h}^{-1}$. Par ailleurs, il existe une composante rapide : sa vitesse est $\geqslant$ à $10 \mathrm{~m} \mathrm{~h}^{-1}$ (Nelson et al., 1958; Penot, 1976).

\section{Direction des transferts}

La circulation dans le phloème est ascendante et descendante. Les mouvements en sens opposés ont lieu dans des faisceaux différents ou dans des tubes criblés différents d'un même faisceau. Une circulation en sens opposés de molécules différentes dans un même tube criblé n'a pu jusqu'alors être prouvée de façon irréfutable.

Chez les arbres, la composante majeure de la circulation libérienne est descendante ; les mouvements libériens ascendants sont localisés et concrétisent le ravitaillement des jeunes pousses ou des fruits par les feuilles les plus proches. Chez le Pommier, il faut par exemple une quinzaine ou une vingtaine de feuilles pour alimenter de facon satisfaisante chaque fruit.

Mécanisme du transport des molécules dans le phloème

Plusieurs théories ont été proposées pour expliquer le transport des molécules dans le phloème. Certaines d'entre elles supposent un mouvement de sève.

\section{Le "mass flow"}

\section{a) Principe}

Il s'agit d'une circulation par pression de turgescence (Münch, 1930). Un modèle simple permet d'illustrer le principe de cette circulation sous pression (Fig. 9). Deux ballons $A$ et $B$ dont la paroi est hémiperméable sont reliés par un tube $T_{1}$. Le ballon $A$ contient une solution concentrée de glucose et le ballon $B$ de l'eau pure. $A$ et $B$ sont placés dans deux bacs $C$ et $D$ reliés par un tube $T_{2}$ et contenant de l'eau pure. L'eau va pénétrer par osmose dans $A$ où la pression hydrostatique augmente. Le liquide de $A$, comprimé, est refoulé vers $B$ qui à son tour envoie de l'eau vers $C$ et $D$. Un circuit est ainsi établi. Celui-ci peut se poursuivre s'il y a apport continu de glucose en $A$ et utilisation continue du glucide en $B$. Ce modèle peut s'appliquer à la circulation des produits de la photosynthèse chez le végétal (Fig. 10). Dans les feuilles, les glucides formés lors de la photosynthèse

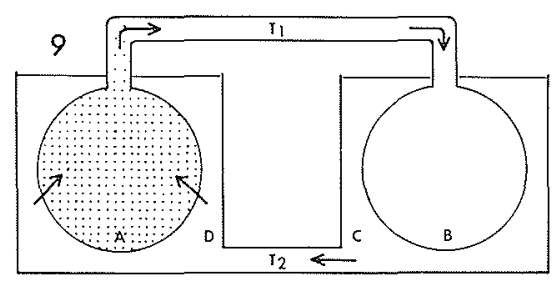

Figure 9 -- Modèle de Münch, légèrement modifié.

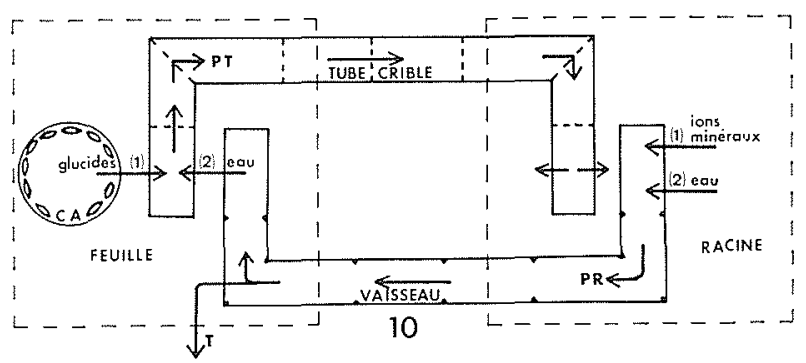

Figure 10 - Modèle possible de la circulation des sèves dans le végétal. Dans la racine, à la suite d'une sécrétion d'ions dans les vaisseaux, il se produit un mouvement osmotique d'eau vers ceux-ci : les vaisseaux sont le siège d'une poussée racinaire (PR). Le jour, à la suite de la transpiration (T), une autre force intervient puissamment pour assurer la montée de la sève "brute" qui est alors sous tension. Dans la feuille, un mécanisme actif assure l'accumulation des glucides dans le complexe cellule criblée-cellule compagne. A la suite d'une entrée osmotique d'eau dans la cellule criblée, le liquide contenu dans les tubes criblés (sève "élaborée") progresse par pression de turgescence (PT). 
s'accumulent dans les nervures. Cette accumulation se fait contre les gradients de concentration : chez le Coton, par exemple, la concentration en glucides simples est 10 fois plus élevée dans les fines nervures que dans les cellules assimilatrices du mésophylle. La pression osmotique s'élève brutalement en passant des cellules du mésophylle bordant la fine nervure ( $8-10$ bars) au complexe cellule criblée-cellule compagne (20-30 bars par exemple). A la suite de cette accumulation de glucides, liée directement ou indirectement à une dépense d'énergie, se produit un afflux d'eau par un processus osmotique, d'où déplacement de la solution, en fait la sève élaborée, par pression de turgescence dans le tube criblé.

\section{b) Quelques arguments}

Le liquide contenu dans les cellules criblées est en général sous pression. Les mesures obtenues suggèrent que la pression de turgescence de ce liquide est souvent comprise entre 10 et 30 bars au niveau des parties aériennes du végétal et qu'un net gradient de pression hydrostatique existe entre le feuillage et les racines, y compris chez les végétaux de petite taille : chez le Ricin, par exemple, ce gradient est voisin de 10 bars (Milburn, 1975). Chez les arbres, des mesures faites suggèrent que le gradient de pression le long du tronc est de l'ordre de 0,4 ou 0,5 bar $\mathrm{m}^{-1}$. Ces mesures sont délicates à réaliser chez le végétal, les agressions physiques (piqûres, incisions, pression) étant susceptibles de modifier le comportement du liber. Les mesures par cryoscopie fournissent le plus souvent des valeurs voisines $(0,35$ bar $\mathrm{m}^{-1}$ chez le chêne, Hammel, 1968) au sujet du gradient de concentration de l'exsudat libérien.

Si l'on étend la loi de Poiseuille aux tuyaux très étroits que sont les tubes criblés, les résultats obtenus

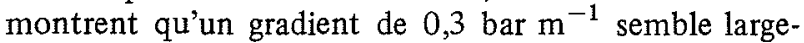
ment suffisant pour permettre un écoulement de sève à la vitesse de $1 \mathrm{~m} \mathrm{~h}^{-1}$ à condition que la lumière et les pores de la cellule criblée soient vides (Weatherley, 1972). Quelques études sur l'ultrastructure des cellules criblées suggèrent que ces conditions sont réalisées chez les Palmiers (Parthasarathy, 1974), des Graminées et, semble-t-il, aussi chez des Dicotylédones (Fig. 8) (Evert et al., 1973).

Les stylets des aphidiens, sectionnés près de la tête de l'insecte alors que ce dernier est "en place" sur la plante, se comportent comme un drain comme nous l'avons déjà mentionné. Un liquide s'écoule pendant longtemps (quelques jours) sans que sa teneur en glucides subisse de modifications importantes. Cela laisse supposer que la cellule criblée est capable de s'approvisionner très rapidement, de façon constante et régulière, à la fois en eau et en glucides. Cette condition est naturellement remplie si elle est le siège d'un déplacement longitudinal de sève.

D'autres observations et d'autres arguments corroborent l'idée de Münch.

\section{c) Critiques}

Un mouvement par pression de turgescence suppose un rôle passif des tubes criblés. Il était donc tentant d'étudier l'action des inhibiteurs et de la température sur le transport libérien.
En général les inhibiteurs du métabolisme $(\mathrm{KCN}$, 2,4-Dinitrophénol) arrêtent la circulation libérienne. Cet effet a été étudié de façon détaillée par Qureshi et Spanner, 1973, qui ont utilisé, comme matériel, les longs stolons de Saxifraga sarmentosa. Les inhibiteurs de la respiration appliqués localement sur le stolon bloquent le transport des produits de la photosynthèse sans entrainer une synthèse de callose au niveau du crible, une réaction possible de la cellule criblée à l'agression chimique, signalée par Ullrich (1963). Il reste cependant à démontrer que ces inhibiteurs appliqués à de fortes concentrations $\left(10^{-2} \mathrm{M}\right.$ à $10^{-3} \mathrm{M}$ pour le DNP), ne modifient pas d'autres aspects de l'intégrité structurale de la cellule criblée. Dans ce contexte, les travaux récents de Giaquinta et Geiger (1977) suggèrent précisément que des inhibiteurs tels que le $\mathrm{KCN}$ bloquent la circulation non pas par action sur des processus métaboliques mais par un effet indirect : à la suite du traitement (KCN à $100 \mathrm{mM}$ ) les pores du crible sont obstrués par des matériaux cytoplasmiques qui présentent habituellement une distribution pariétale chez les témoins.

Les inhibiteurs de la protéosynthèse, comme la cycloheximide, bloquent aussi les translocations dans le liber (Penot, 1977) : l'idée d'une relation entre synthèse protéique et transport, plus précisément entre $P$-protéine et circulation libérienne a donc été avancée.

Les basses températures peuvent aussi affecter cette circulation entraînant notamment l'arrêt du transport au voisinage de $0^{\circ} \mathrm{C}$. Mais cet effet n'est pas général. Chez. Salix viminalis le transport libérien s'arrête seulement lorsque la sève gèle, c'est-à-dire à $-4^{\circ} \mathrm{C}$. Des "applications" locales de froid sur la tige $\left(0^{\circ} \mathrm{C}\right.$ ou $-2^{\circ} \mathrm{C}$ ) ne semblent pas affecter la circulation libérienne alors que la respiration des tissus est considérablement diminuée : à $-2^{\circ} \mathrm{C}$, celle-ci n'atteint que $5 \%$ de sa valeur à $25^{\circ} \mathrm{C}$ (Weatherley et Watson, 1969).

Selon d'autres hypothèses, les cellules criblées ellesmêmes jouent un rôle actif facilitant ou même contrôlant totalement la translocation.

\section{Progression de sève facilitée ou due à des mouvements rythmiques du matériel protéique}

\section{a) Principe}

$a_{1}$ ) Selon Thaine (1969) les tubes criblés sont occupés par des "cordons transcellulaires" qui traversent les pores et qui s'étendent d'une cellule criblée à l'autre. Ces "cordons" (Fig. 11), dont le diamètre chez Cucurbita est d'environ $5 \mu \mathrm{m}$, sont limités par une enveloppe constituée de filaments protéiques situés entre $2 \mathrm{mem}$ branes (Thaine, 1973). A l'intérieur de l'enveloppe on trouve un certain nombre de tubules. La sève contenue dans les tubules se déplace à la suite de contractions rythmiques de l'enveloppe protéique. Il y a, en quelque sorte, propagation de vagues péristaltiques le long des cordons transcellulaires (Fig. 11 et 12).

$a_{2}$ ) Selon Fensom (1975), la circulation de la sève dans le tube criblé pourrait être assurée ou facilitée par des mouvements ondulatoires de filaments protéiques inserrés soit sur la paroi ou dépendant, par une extrémité, d'un faisceau de filaments (Fig. 13). Par ailleurs, des "pulses" (Fig. 14) peuvent se propager dans un sens ou dans l'autre, indépendamment du mouve- 


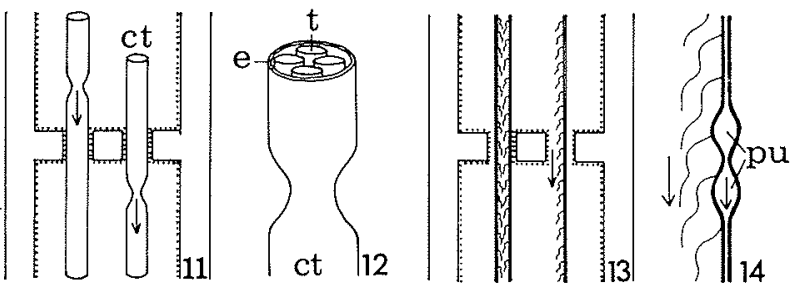

Figures 11 et 12 - Modèle de Thaine (1969-1973), légèrement modifié (ct, cordons transcellulaires ; e, enveloppe protéique contractile; $t$, tubule).

Figures 13 et 14 - Modèle de Fensom et Williams, 1974 (cf. Fensom, 1975), légèrement modifié (pu, pulse)

ment de liquide facilité par les mouvements ondulatoires. Ces "pulses" résulteraient du resserrement puis de l'éloignement des composantes des tubules.

\section{b) Quelques arguments}

Ces modèles supposent une dépense d'énergie tout le long du conduit. Or la cellule criblée contient des quantités appréciables d'ATP $(100$ à $300 \mu \mathrm{g} / \mathrm{ml}$ d'exsudat) provenant probablement des cellules compagnes. Ainsi pourrait éventuellement s'expliquer l'action des inhibiteurs de la respiration.

\section{c) Critiques}

En microscopie photonique on peut voir des structures apparemment tubulaires s'étendant d'un crible à l'autre mais les cordons transcellulaires n'ont jamais été observés en microscopie électronique. L'ontogenèse de ces formations complexes est difficilement explicable. Enfin, les modèles de Thaine (1969) et de Fensom (1974) supposent des propriétés particulières d'une fraction au moins du matériel protéique de la cellule criblée. Les propriétés des " $P$-protéines" ont donc fait l'objet d'une étude relativement détaillée. Ce matériel ne se lie pas à la colchicine et ne présente pas d'activité ATP-asique in vitro; la cytochalasine $B$, qui bloque la cyclose, n'arrête pas le transport libérien (Williamson, 1972) : les protéines de l'exsudat qui se caractérisent par leur forte teneur en lysine n'ont donc pas, en définitive, les propriétés de l'actine ou de la tubuline. En Outre, elles se caractérisent aussi par leur diversité : les 4 protéines basiques (PM : $30000,60000,116000$ et 220000) de l'exsudat libérien de Cucurbita maxima (Beyenbach et al., 1974) ne semblent apparemment pas avoir de parenté avec la composante protéique majeure observée chez le Ricin (Sloan et al., 1976).

Finalement, la seule information pour l'instant favorable à l'idée de propriétés particulieres des " $P$ protéines" de la cellule criblée est celle fournie par Ilker et Currier (1974). Ils font incuber des sections de tige de Fève dans une solution contenant de la méromyosine $\mathrm{H}$. De même qu'il y a formation d'un complexe avec l'actine, il y a ici, selon les auteurs, formation d'un complexe avec le matériel protéique de la région du crible.

\section{Conclusion}

Seuls quelques faits ayant une relation avec la possibilité d'une circulation de sève ont été brièvement présentés. Cependant d'autres résultats laisseraient plutôt croire à une relative immobilité de l'eau dans le tube criblé et, en particulier, à l'existence d'un processus de diffusion de type interfacial : déplacements rapides de petites quantités de matière, effet parfois nul des basses températures sur la vitesse de transport alors que, selon l'équation de Poiseuille, l'augmentation de la viscosité devrait entraîner un ralentissement de transport s'il y avait circulation de sève. Ce processus de diffusion apparait comme une composante possible du transport libérien (Penot, 1976).

Peut-on parler d'une circulation de sève dans les tubes criblés ? vraisemblablement oui chez certains groupes végétaux comme les Palmiers. Chez les Dicotylédones, un écoulement est une composante très probable du transport libérien puisque des virus et des mycoplasmes se trouvent entraînés dans les régions en voie de croissance. Un mouvement de liquide a même été visualisé à l'aide de particules de carbone dans des cordons de phloème isolé de l'Heracleum (Barclay et Fenson, 1973) ; cependant la vitesse d'écoulement observée, voisine de $1 \mathrm{~cm} \mathrm{~h}^{-1}$, est considérablement inférieure à celle de la circulation libérienne classique. Le matériel végétal, préparé pour l'expérience, n'a-t-il pas réagi aux agressions, masquant ainsi ses potentialités ? Il convient de souligner une nouvelle fois que la sensibilité du végétal, une propriété trop souvent ignorée, et l'extrême fragilité de la cellule criblée compliquent sans cesse la tâche de l'expérimentateur s'intéressant à la physiologie du phloème. Par ailleurs, le faisceau d'arguments en faveur de l'existence d'un transport par pression de turgescence chez divers groupes de plantes supérieures ne permet pas d'exclure, ipso facto, la manifestation d'autres mécanismes dans les autres groupes. Aussi les recherches entreprises sur les Algues (Penot et al., 1976), végétaux chez lesquels l'appareil conducteur peut présenter une esquisse de différenciation, sont-elles particulièrement dignes d'intérêt. Enfin, la démonstration éventuelle d'un transport par pression de turgescence chez un végétal donné ne permet pas d'exclure l'intervention concomitante de processus diffusifs, notamment d'un mouvement de type interfacial.

\section{Bibliographie}

BARCLAY G.F, et FENSOM D.S., (1973) - Passage of carbon black through sieve plates of unexcised Heracleum sphondylium after micro injection, Acta Bot. Neerl, 22, 228-232

BEYENBACH J., WABER C. et KLEINIG H., (1974) - Sieve tube proteins from Cucurbita maxima, Planta, 119, 113-124.

CRONSHAW J. et ESAU K. (1967) - Tubular and fibrillar components of mature and differentiating sieve elements, $J$. Cell. Biol., 34, 801-815.

CRONSHAW J, et ESAU K., (1968). - P-protein in the phloem of Cucurbita. I. The development of P-protein bodies, $J$. Cell. Biol., 38, 25-39.

DIXON H.H., (1922). - Transport of organic substances in plants, Nature, 110, 547-551. 
EVERT R.F., ESCHRICH W. et EICHHORN S.E., (1973). P-protein distribution in mature sieve elements of Cucurbita maxima, Planta, 109, 193-210.

FENSOM D.S., (1975). - Other possible mechanisms, in "Transport in plants I Phloem transport" Ed. Zimmermann M.H. and Milburn J.A., Springer Verlag, Berlin, Heidelberg, NewYork, 354-366.

FISHER D.E., (1975). - Structure and functional soybean sieve elements, Plant Physiol., 56, 555-569.

GIAQUINTA R. et GEIGER D.R., (1977). - Mechanism of cyanide inhibition of phloem translocation, Plant Physiol., $59,178-180$.

HAMMEL H.T., (1968). - Measurement of turgor pressure and its gradient in the phloem of oak, Plant Physiol., 43, 10421048.

ILKER R. et CURRIER H.B., (1974). - Heavy Meromyosin complexing filaments in the phloem of Vicia faba and xylosma congestum, Planta, 120, 311-316.

MILBURN J.A., (1975) - Pressure flow, in "Transport in plants I. Phloem transport" Ed. Zimmermann M.H. and Milburn J.A., Springer-Verlag, Berlin, Heidelberg, New-York, 328353.

MUNCH E. (1930). - Die Stoffbewegungen in der Pflanze, Fisher, lena

NELSON C.D., PERKINS H.J. et GORHAM P.R., (1958). Note on a rapid translocation of photosynthetically assimilated ${ }^{14} \mathrm{C}$ out of the primary leaf of the young soybean plant, Can. J. Biochem. Biophys., 36, 1277-1279.

NUSKE J et ESCHRISCH W., (1976). - Synthesis of P-protein in mature phloem of Cucurbita maxima, Planta, 132, 109118.

PARTHASARATHY M.V., (1974). - Ultrastructure of phloem in palms. III. Mature phloem, Protoplasma, 79, 265-315.
PENOT M., (1976). - Mise en évidence de déplacement de ${ }^{86} \mathrm{Rb}$ à grande vitesse, dans le phloème de Tradescantia viridis, Planta, 132, 183-187.

PENOT M., (1977). - De l'influence de la cycloheximide sur l'absorption foliaire et sur la redistribution par le phloème de quelques éléments minéraux, Physiol, vég., 15, 133-144.

PENOT M., FLOC'H J.Y. et PENOT M., (1976). - Physiologie comparée des transports à longue distance chez les végétaux supérieurs et chez les algues marines, Phycologia, 15, 299-308.

QURESHI F.A. et SPANNER D.C., (1973). - The influence of dinitrophenol on phloem transport along the stolon of Saxifraga sarmentosa, Planta, 111, 1-12.

SLOAN R T., SABNIS D.D. et HART J.W., (1976). - The hetetogeneity of phloem exudate proteins from different plants : a comparative survey of ten plants using polyacrylamide gel electrophoresis, Planta, 132, 97-102.

THAINE R., (1969). - Movement of sugars through plants by cytoplasmic pumping, Nature, 222, 873-875.

THAINE R., (1973). - Transcellular strands of cytoplasm in sieve tubes of Squash, Nature, 245, 161-163.

ULLRICH W., (1963). - Uber die Bildung von Kallose bei einer Hemmung des Transportes in den Siebröhren durch Cyanid, Planta, 59, 387-390.

WEATHERLEY P.E. (1972) - Translocation in sieve tubes : some throughts on structure and mechanism, Physiol. vég. 10, 731-742.

WEATHERLEY P.E. et WATSON B.T. (1969) - Some lowtemperature effects on sieve tube translocation in Salix viminalis, Ann. Bot., 33, 845-853.

WILLIAMSON R.E. (1972) - An investigation of the contractile protein hypothesis on phloem translocation, Planta, $106,149-157$

\title{
Discussion
}

\author{
Président : M.S. HENIN
}

M. le Président remercie M. BONNEMAIN et il ouvre la discussion.

M. COMOLET demande si le gradient de pression suffit à justifier la circulation des cellules criblées et s'il ne convient pas d'introduire des mouvements péristaltiques.

M. BONNEMAIN indique qu'il y a vraisemblablement mouvement par pression de turgescence chez certaines espèces, cela supposant un rôle passif de la cellule criblée entre l'organe donneur, la feuille, et l'organe receveur, la racine.

Le Président évoquant des vitesses "impressionnantes" de l'ordre de $10 \mathrm{~m} / \mathrm{h}, \mathrm{M}$. BONNEMAIN signale que l'on a vu des vitesses de 50 ou $60 \mathrm{~m} / \mathrm{h}$.

Il résume ensuite les travaux menés par LEE sur la structure et la rôle de la cellule criblée.

LEE a donc évalué le nombre des filaments protéiques contenus đans la cellule criblée. Il est arrivé, je crois, à 2 ou $3.10^{5}$. Compte tenu de leur diamètre, de l'ordre de 100 ou 200 angströms, il a calculé leur surface. Compte tenu des dimensions de la cellule criblée, de la dimension des molécules de saccharose et du fait qu'il y a environ $10 \%$ de saccharose dans ce type cellulaire, il a estimé le nombre de molécules de saccharose contenues dans une cellule criblée. Il a calculé également le nombre de molécules portées par l'ensemble des filaments protéiques. Les deux chiffres obtenus ne sont pas très différents, ce qui peut signifier que ces filaments peuvent porter, en couche monomoléculaire, une bonne partie des molécules de saccharose se trouvant dans la cellule criblée. Il se base également sur un travail de VAN DEN HONERT datant de 1932. Lorsqu'on introduit au niveau de la surface de séparation des liquides non miscibles (eau et éther) de l'oléate de potassium par exemple, on constate alors que la substance introduite a tendance à se disposer en couche monomoléculaire. LEE suppose done qu'il y aurait glissement des molécules de saccharose le long des filaments de protéine-P. Cela expliquerait en particulier les vitesses très grandes que l'on observe parfois, car il est difficile d'admettre qu'elles soient dues à un mouvement de liquide. Par ailleurs, l'inhibition du transport par les inhibiteurs de la synthèse protéique peut à la rigueur être considérée comme un argument en faveur de la conception de LEE.

Le Président compare ce phénomène à une sorte de tapis roulant sur lequel seraient fixées les molécales, le tapis étant tiré.

Sur une question de M. KOPP, M. BONNEMAIN précise, dans le cadre de l'hypothèse de LEE, que le tapis moléculaire serait "tiré" par les organes receveurs.

M. KOPP mentionne les phénomènes de transport observés dans la trachée artère mais en soulignant qu'ils sont très différents : les couches sont beaucoup plus épaisses et le transport peut être mis en évidence de façon tout à fait macroscopique.

Répondant à une question de M. VERNET relative à l'utilisation de l'eau tritiée pour l'étude de l'écoulement, $M$ BONNEMAIN souligne combien cette méthode est délicate. L'expérience montre en effet qu'il y a des migrations extrèmement importantes d'eau tritiée vers les tissus adjacents. 